EUROPHYSICS LETTERS

Europhys. Lett., (), pp. ()

\title{
On the High-Pressure Phase Transition in $\mathrm{GaPO}_{4}$
}

\author{
James Badro $^{1,2}$, Jean-Paul Itié ${ }^{2}$ and Alain Polian ${ }^{2}$ \\ 1 Laboratoire de Sciences de la Terre, École normale supérieure de Lyon \\ 46, allée d'Italie 69364 Lyon Cedex 07, France. \\ 2 Physique des Milieux Condensés, Université Pierre et Marie Curie, Bry \\ 4, place Jussieu 75252 Paris Cedex 05, France. \\ (received ; accepted ) \\ PACS. $62.50+\mathrm{p}-$ High Pressure. \\ PACS. 61.50.Ks - Phase Transitions. \\ PACS. 61.10-i - X-ray diffraction.
}

\begin{abstract}
X-ray diffraction (XRD) experiments have been carried out on quartz-like $\alpha-\mathrm{GaPO}_{4}$ at high pressure and room temperature. A transition to a high pressure disordered crystalline form occurs at $13.5 \mathrm{GPa}$. Slight heating using a YAG infrared laser was applied at $17 \mathrm{GPa}$ in order to crystallize the phase in its stability field. The structure of this phase is orthorhombic with space group $\mathrm{Cmcm}$. The cell parameters at the pressure of transition are $a=7.306 \AA, b=5.887 \AA$ and $c=5.124 \AA$.
\end{abstract}

Introduction. -

The low pressure polymorphs of quartz and quartz like compounds are made up of tetrahedrally coordinated cations. The high pressure structures consist of octahedral cation arrangements, in which oxygen atoms are packed in a body-centered cubic (bcc) sub-lattice around the cations [1]. At room temperature, the kinetics of these four- to six-coordinated transitions is very slow leading to a pressure-induced amorphization (PIA) process observed in quartz [2, 3, 田, 5, 6, 1. The compression at room temperature of isostructural compounds $\alpha-\mathrm{GeO}_{2}$ and $\alpha-\mathrm{GaAsO}_{4}$ has also been studied by X-ray diffraction and extended X-ray absorption fine structure (EXAFS) spectroscopy [7, 8, 9] and the studies show that cation coordination rises from four to six through the transition.

In the case of berlinites, it is now established that $\alpha-\mathrm{AlPO}_{4}, \alpha-\mathrm{AlAsO}_{4}, \alpha-\mathrm{GaPO}_{4}$ and $\alpha-\mathrm{GaAsO}_{4}$ exhibits a polymorphic crystalline phase transition, the high pressure form being very poorly crystallized, and therefore yielding very poor X-ray diffraction patterns [10, 11, 12, 9, 13]. In-situ EXAFS experiments were realized on $\alpha-\mathrm{GaPO}_{4}$ at the K-edge absorption energy of gallium 11, 13] and the polymorphic phase transition observed at $12 \mathrm{GPa}$ is associated with a four- to six-fold oxygen coordination change observed around gallium atoms. 
Molecular dynamics (MD) simulations on berlinite $\left(\alpha-\mathrm{AlPO}_{4}\right)$ associate the transition with a de-stabilization of the $\mathrm{AlO}_{4}$ tetrahedron and its transition towards an $\mathrm{AlO}_{6}$ octahedron 114. 15]; they also have concluded that room temperature PIA would occur only when the $\mathrm{PO}_{4}$ tetrahedra went unstable, at a predicted pressure of $80 \mathrm{GPa}$, this pressure having not yet been produced experimentally on $\mathrm{AlPO}_{4}$.

\section{Experimental. -}

Quartz structured gallium phosphate single crystals grown by the hydrothermal technique were crushed into powder and used for this high pressure energy dispersive X-ray diffraction study. Platinum powder was mixed with the sample in order to absorb the infrared beam emmited by a YAG laser and heat the system. The mixture was then loaded in a stainless steel gasket with a $250 \mu \mathrm{m}$ hole diameter and $40 \mu \mathrm{m}$ initial thickness and high pressure was generated by a membrane driven [16] diamond anvil cell (DAC). Nitrogen was used as pressure transmitting medium, and ruby fluorescence as well as platinum isothermal equation of state (EOS) were used as pressure gauges; The measurements were carried out on the DW-11 energy dispersive X-ray diffraction beamline on the DCI ring of the LURE synchrotron facility in Orsay, France. Accumulation times ranged from 1 hour at low pressure and up to 12 hours in the high pressure phase with a diffraction angle $2 \theta=12^{\circ}$. At $17 \mathrm{GPa}$, the sample was heated by diffusion of heat from the infrared absorbing platinum mixed with the sample.

Compression and Heating. -

The high-pressure behaviour of the quartz phase of $\mathrm{GaPO}_{4}$ has been studied by X-ray diffraction [17, 13, 11], Raman [18, 13] and Brillouin 13] scattering.

Isothermal equations of state (EOS) have been fitted on pressure-volume data and yield similar results. As with other berlinite phases, it was assumed that $\mathrm{GaPO}_{4}$ transformed to an amorphous phase [17] at $9 \mathrm{GPa}$, but new experiments [13, 11] show that this transformation is instead due to a polymorphic crystalline transition.

In this experiment, very few points were produced at low pressure. At a pressure of $17 \mathrm{GPa}$, the sample had already crossed the transition pressure and the spectrum of the high-pressure phase is reproduced in figure 1 . It was impossible to index the peaks due to both the disorder of the phase and the broadening induced by the very small size of the crystallites in the high pressure phase after such thermally inhibited reconstructive phase transitions. At this point, the sample was heated in order to "activate" the kinetics of the transition. Care was taken in order to insure that no phase transition occurred during heating. The system was brought back to ambient temperature and a diffraction spectrum was collected. The sample was very well crystallized and the diffraction lines (figure 1) can be indexed in an orthorhombic system (table I) with space group $\mathrm{C}_{\mathrm{mcm}}$, and the phase is of $\mathrm{InPO}_{4}$ structure-type, with octahedral gallium and tetrahedral phosphorus atoms [11, 9, 13]. At this pressure, the unit cell parameters were $a=7.306(7) \AA, b=5.887(6) \AA$ and $c=5.124(3) \AA$. A detailed analysis of these two spectra shows that no transition occurred during heating, because none of the existing diffraction lines before heating had vanished afterwards, and most of the new line which appeared after heating were already present before but were broad and very weak (figure il). It is only after such an analysis that we could infer that these two spectra are that of the same thermodynamic phase, one of which is much better crystallized than the other. It can be noted here that this is the first characterization, though indirect, of the high-pressure and room-temperature phase of $\alpha-\mathrm{GaPO}_{4}$.

It is interesting to note that this phase is similar to the one obtained by room temperature compression of a high-temperature polymorph of $\mathrm{GaPO}_{4}$, namely the low cristobalite phase [19, 20]. This finding confirms that the transition of the quartz phase at high pressure is thermally inhibited because cristobalite being metastable at room temperature, it has a higher 
free enthalpy than the quartz-form and therefore it necessarily has a lower thermodynamic energy barrier with respect to the high pressure orthorhombic phase, as well as a higher driving force for the transition. It can therefore transit to this structure without the need for kinetic energy activation brought by heating.

The system was then further compressed in order to measure the bulk modulus of the new high pressure phase. Three points at higher pressure were undertaken, and a Birch-Murnaghan third order equation of state was used for fitting through the points (figure 2). The pressure derivative of the bulk modulus was fixed at $\mathrm{K}_{0}^{\prime}=4$, the unit cell volume at ambient pressure was found to be $\mathrm{V}_{0}=231.7 \AA^{3}$ and the fitted bulk modulus equal to $\mathrm{K}_{0}=308 \mathrm{GPa}$.

\section{Decompression. -}

A step by step decompression path was then followed in order to have a precise view on the back-transformation of the sample. The high pressure phase is retained down to $6 \mathrm{GPa}$. At $1 \mathrm{GPa}$, the (200) peak of $\alpha-\mathrm{GaPO}_{4}$ along with other weak peaks appear in the diffraction pattern, together with the peaks of the high pressure orthorhombic phase. Thus, the quenched sample consists of a mixture of both phases, meaning the the transition is not fully reversible. It should be noted that these points obtained on decompression that were still in the high pressure phase were not included in the fit for the EOS, because a huge amount of stress appears at downstroke, and the points do not generally coincide with those obtained at compression as can be seen at $18.5 \mathrm{GPa}$ in figure 2 .

\section{Conclusion. -}

High-pressure heating experiments have been undertaken and show that an increase in the temperature enhances dramatically the crystallization of the sample in the high-pressure phase, thus yielding narrower peaks. The phase was then indexed in an orthorhombic symmetry in the $\mathrm{Cmcm}$ space group. The high pressure phase is of $\mathrm{InPO}_{4}$ type, with octahedral gallium and tetrahedral phosphorus. The high pressure phase obtained seems to be identical to the high pressure crystal formed by the room temperature compression of the high temperature polymorph. This seems to confirm the fact that the transition from the $\mathrm{P} 3{ }_{1} 21$ quartz-like material to the $\mathrm{Cmcm}, \mathrm{InPO}_{4}$-like phase is thermally inhibited at room temperature. Even though this transition does take place in the stability field of the phase, room temperature kinetics are not sufficient to drive the transition. This confirms that the high pressure phase transition in $\mathrm{GaPO}_{4}$ is a polymorphic crystalline phase transition and not a pressureinduced amorphization, as previously reported [17]. The observed gallium four-fold to six-fold coordination change at room temperature is therefore associated with the expected quartzto- $-\mathrm{InPO}_{4}$ transformation.

A similar transformation occurs in $\alpha-\mathrm{AlPO}_{4}$ at room temperature, at least from the spectroscopic point of view, and these two materials, i.e. $\mathrm{AlPO}_{4}$ and $\mathrm{GaPO}_{4}$, exhibit a similar high-pressure behaviour. It has very recently been shown that isostructural $\mathrm{FePO}_{4}$ undergoes a similar transformation to the $\mathrm{InPO}_{4}$ structure 21] at high pressure. This study shows that it is therefore likely that the $\mathrm{InPO}_{4}$ phase is the host structure for phosphate berlinites at high pressure.

In this transformation, the phosphorus tetrahedra remain unaltered. At room-temperature, the partial back-transformation to the quartz-phase is a mirror of the high-pressure transformation. The back-transformation associated with a conservation of the crystallographic axes is a direct consequence of the rigidity of these $\mathrm{PO}_{4}$ clusters.

We wish to express our grateful thanks to our colleagues Fabrice Visocekas and Denis Andrault of the Dpartement des Gomatriaux at the Institut de Physique du Globe de Paris for carrying out the laser heating of the sample. We sincerely acknowledge Philippe Gillet for his comments and discussions on this work.

Laboratoire de Sciences de la Terre is CNRS UMR 5570. Physique des Milieux Condensés is CNRS URA 782. 


\section{REFERENCES}

[1] P.J. Heaney, C.T. Prewitt, and G.V. Gibbs, editors. Silica. Physical Behavior, Geochemistry and Materials Applications, volume 29 of Reviews in Mineralogy. Mineralogical Society of America, 1994.

[2] R.J. Hemley. In High-Pressure research in mineral physics, Mineral Physics 2. Terra Scientific Publishing Company - AGU, 1987.

[3] R.J. Hemley, A.P. Jephcoat, H.K. Mao, L.C. Ming, and M.H. Manghnani. Nature, 334:52-54, 1988.

[4] C. Meade, R.J. Hemley, and H.K. Mao. Phys. Rev. Lett., 69(9):1387-1390, 1992.

[5] L.E. McNiel and M. Grimsditch. Phys. Rev. Lett., 68:83-85, 1992.

[6] K.J. Kingma, C. Meade, R.J. Hemley, H. Mao, and D.R. Veblen. Science, 259:666-669, 1993.

[7] J.-P. Iti, A. Polian, G. Calas, J. Petiau, A. Fontaine, and H. Tolentino. Phys. Rev. Lett., 63(9):389-401, 1989.

[8] J. Badro, Ph. Gillet, P.F. McMillan, A. Polian, and J.-P. Iti. Europhys. Lett., in press.

[9] J. Badro, J.-P. Iti, A. Polian, and Ph. Gillet. J. Physique IV, 7:C2-987, 1997.

[10] Ph. Gillet, J. Badro, B. Varrel, and P.F. McMillan. Phys. Rev. B, 51(17):11262-11269, 1995.

[11] J.-P. Iti, T. Tinoco, A. Polian, G. Demazeau, S. Matar, and E. Philippot. High Pressure Research, 14:269-276, 1996.

[12] H. Sowa and H. Ahsbahs. Zeit. für Krist., (211):96-100, 1996.

[13] A. Polian, J.-P. Iti, and J. Badro. Unpublished, .

[14] J.S. Tse and D.D. Klug. Science, 255:1559-1561, 1992.

[15] G.W. Watson and S.C. Parker. Phys. Rev. B, 52:13306-13309, 1995.

[16] R. LeToullec, J.-P. Pinceaux, and P. Loubeyre. High Press. Res., 1:77-90, 1988.

[17] H. Sowa. Zeit. für Krist., 209:954-960, 1994.

[18] M. Verhelst, J. Hu, and G.H. Wolf. Private Communication.

[19] J.L. Robeson, R.R. Winters, and W.S. Hammack. Phys. Rev. Lett., 73(12), 1994.

[20] V.V. Murashov, L.S. Dubrovinsky, J.S. Tse, and Y. LePage. J. Phys. Condens. Matter, 7:8279, 1995.

[21] M.P. Pasternak, G.Kh. Rozenberg, E. Milner, M. Amanowicz, T. Zhou, U. Schwarz, K. Syassen, R. Dean Taylor, M. Hanfland and K. Brister. Phys. Rev. Lett., 79:4409, 1997. 


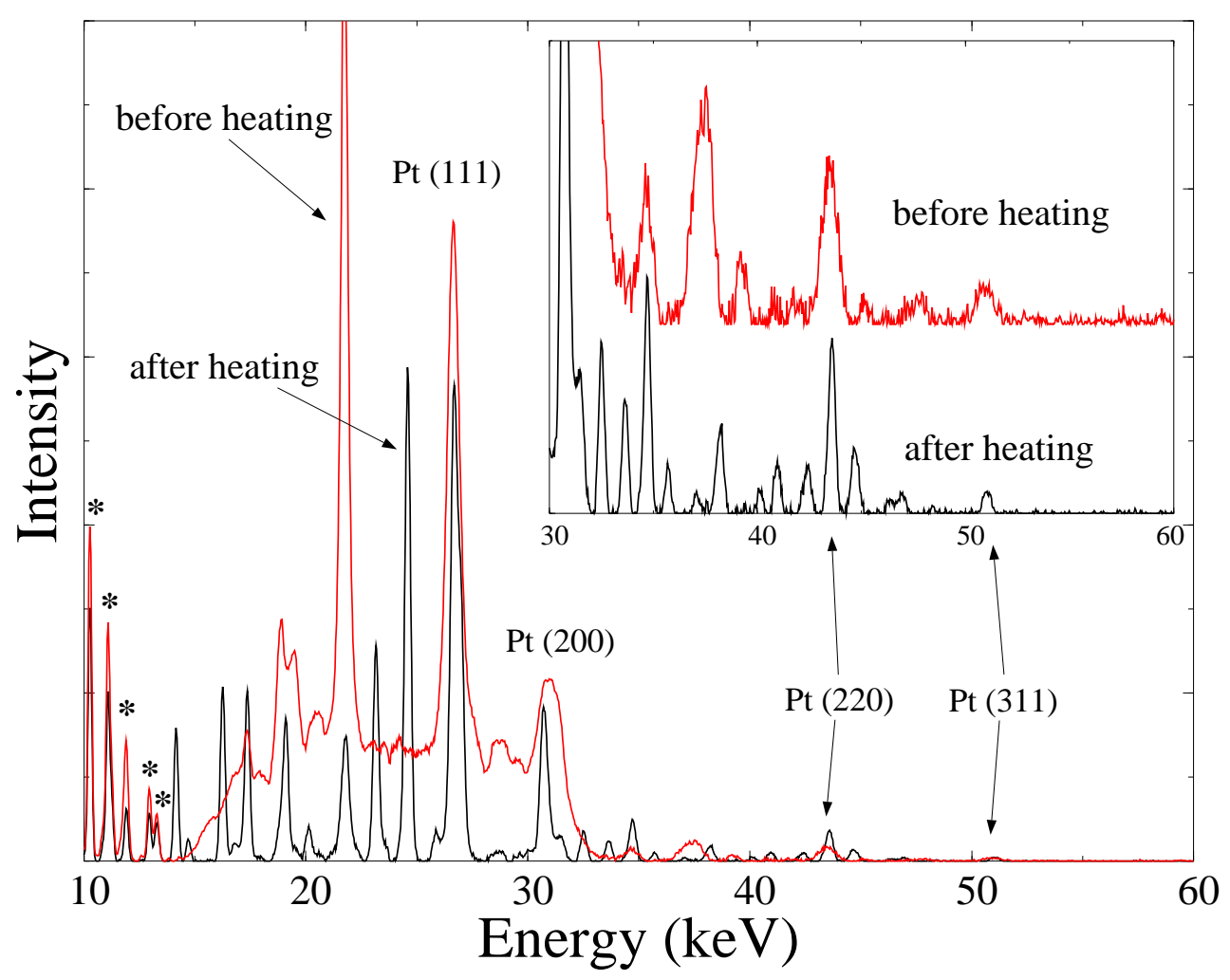

Fig. 1. - X-ray diffraction spectra of $\mathrm{GaPO}_{4}$ at $17 \mathrm{GPa}$ before and after laser heating. The heated sample exhibits a finer crystalline state, and care was taken in order to ensure no phase transition occured upon heating. Gallium and platinum fluorescence lines are marked with a star $\left(^{*}\right)$ sign (in the $10-13 \mathrm{keV}$ range). Platinum diffraction peaks are also indicated on the figure, along with the corresponding Miller indices. The inset shows a detailed view of the spectra in the high energy domain and we observe that most of the "new" diffraction lines were already present, but were broad and weak. 
EUROPHYSICS LETTERS

TABLE I. - Observed and calculated (hkl) spacings for the high pressure phase at 17 GPa after heating. The calculated lines are that of an orthorhombic system with a centered Bravais lattice. The cell parameters are $a=7.306 \AA, b=5.887 \AA$ and $c=5.124 \AA$. The difference between observed and calculated spacings is taken using 5 decimal places for observed lines, but the latter can reasonable de given with only three significant digits.

\begin{tabular}{|c|c|c|c||c|c|c|c|}
\hline$(h k l)$ & $d_{\text {obs }}(\AA)$ & $d_{c a l}(\AA)$ & $d_{o b s}-d_{c a l}(\AA)$ & $(h k l)$ & $d_{o b s}(\AA)$ & $d_{c a l}(\AA)$ & $d_{\text {obs }}-d_{c a l}(\AA)$ \\
\hline 101 & 4.195 & 4.19543 & -0.00026 & 200 & 3.650 & 3.65313 & -0.00360 \\
111 & 3.419 & 3.41677 & 0.00211 & 210 & 3.107 & 3.10419 & 0.00272 \\
020 & 2.946 & 2.94397 & 0.00207 & 120 & 2.725 & 2.73063 & -0.00601 \\
211 & 2.661 & 2.65506 & 0.00567 & 002 & 2.562 & 2.56226 & -0.00064 \\
102 & 2.414 & 2.41789 & -0.00402 & 121 & 2.413 & 2.40986 & 0.00401 \\
301 & 2.198 & 2.19965 & -0.00160 & 130 & 1.888 & 1.89545 & -0.00717 \\
400 & 1.825 & 1.82657 & -0.00169 & 302 & 1.764 & 1.76524 & -0.00149 \\
003 & 1.710 & 1.70817 & 0.00186 & 103 & 1.662 & 1.66332 & -0.00109 \\
113 & 1.600 & 1.60068 & -0.00091 & 023 & 1.479 & 1.47748 & 0.00138 \\
123 & 1.449 & 1.44816 & 0.00094 & 303 & 1.400 & 1.39848 & 0.00166 \\
422 & 1.328 & 1.32753 & 0.00030 & & & & \\
\hline
\end{tabular}




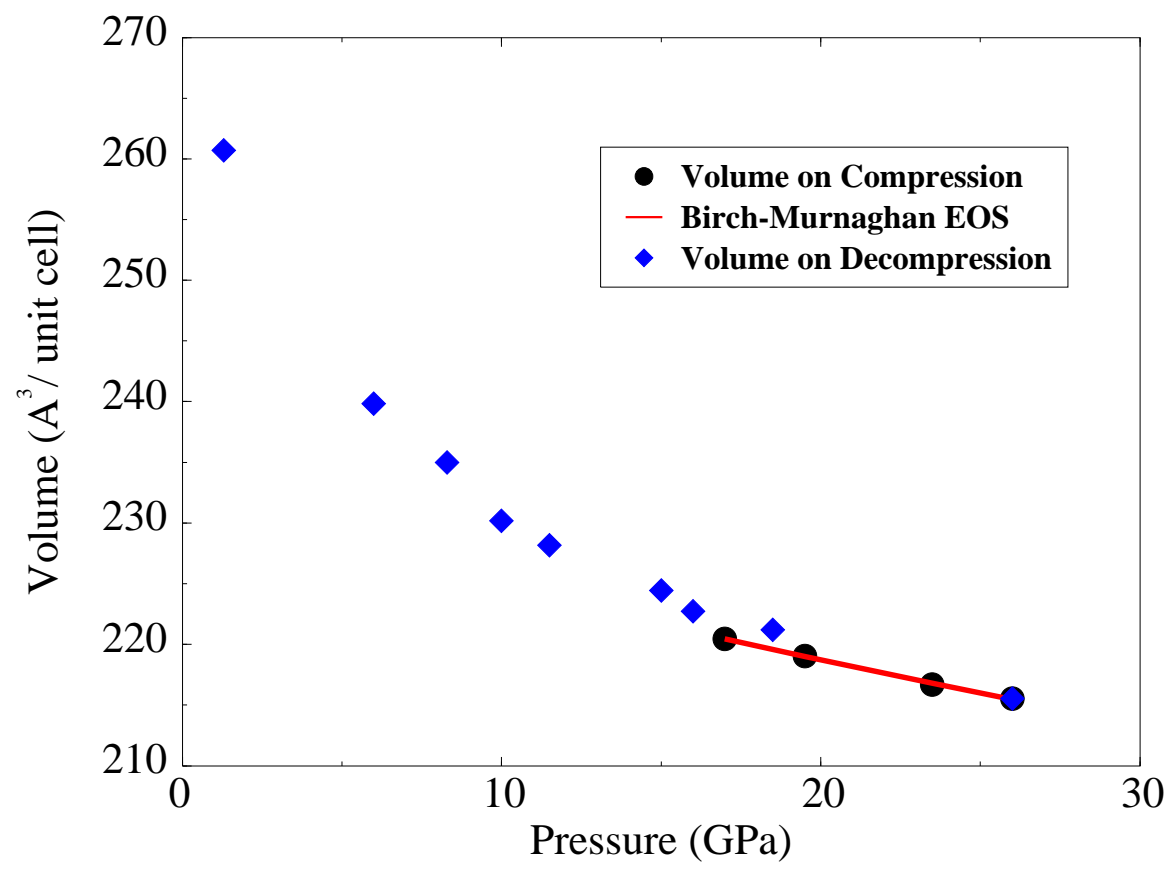

Fig. 2. - Pressure-Volume data points obtained from refinement of the orthorhombic structure of the sample in the high pressure phase. The points are fitted by a Birch-Murnaghan third order EOS. Observed values for the variables are: $K_{0}=308 \mathrm{GPa}$ and $\mathrm{V}_{0}=231.7 \AA^{3}$, with the pressure derivative of the bulk modulus set to 4. This equation is fitted with four points (filled circles) from $17 \mathrm{GPa}$ up to $26 \mathrm{GPa}$. The points on decompression (filled diamonds) were not used, because the apparition of deviatoric and uniaxial stresses in the sample makes volume data unreliable (for example, point at 18.5 GPa on decompression). 